\title{
CME quiz
}

\section{A complete set of instructions for the first-time smoker.}

Don't.

American Heart Association

WE'RE FIGHTING FOR YOUR LIFE

\section{The purpose of this quiz is to} provide a convenient means of self-assessment of your reading of the scientific content of this issue of JAOA. Enter your answers to the questions in the spaces provided so that you can easily check them with the answers that will be published next month.

To apply for CME credit, transfer your answers to the mail-in card on page 41 and return it to the CME office. So that you may complete this self-assessment in privacy, use only your member number to apply for $\mathrm{CME}$ credit. The CME office will record only the fact that you have completed the self-assessment test. Any grading will be done by the Editorial Department only for the purpose of planning areas of study which may be helpful to cover in future issues of JAOA.
1. The incidence of thrombocytopenia during low-dose heparin prophylaxis is

(a) 0 percent.

(b) 3 percent.

(c) 10 percent.

(d) 30 percent

(e) 50 percent.

2. During low-dose heparin therapy,

(a) platelet counts should be obtained daily.

(b) platelet counts should be obtained every third day.

(c) platelet counts should be obtained if petechiae or clinical bleeding occur.

(d) platelet counts are not required.

3. Concerning a lacrimal gland cyst, which of the following statements are true?

(a) It arises from a lacrimal gland duct.

(b) It can be identified on CT as a cystic mass adjacent to the lacrimal gland and with no associated bony changes.

(c) It is treated by surgical removal.

(d) It is a bluish mass which projects into the superotemporal aspect of the orbit from the lacrimal fossa region.

(e) All of the above statements are true.

4. Paraduodenal hernia accounts for what percentage of all cases of bowel obstruction?

(a) Less than 1 percent

(b) 5 percent

(c) 15 percent

(d) 50 percent

5. The clinical signs and symptoms 
associated with a paraduodenal hernia includes which one of the following options?

(a) Fever and hyperbilirubinemia

(b) Vomiting and large bowel obstruction

(c) A history of chronic abdominal pain and recurrent small bowel obstruction

6. The pathognomonic radiographic appearance of a paraduodenal hernia is

(a) a gasless abdomen.

(b) loops of small bowel filled with fluid or contrast material, confined to one area of the abdomen and enclosed within a sac. (c) a persistent air fluid level in the cecum.

(d) an abnormal small bowel mucosal pattern and fistula formation.

7. Vibrio alginolyticus has been im- implicated in all of the following diseases except

(a) otitis externa.

(b) gastroenteritis.

(c) cellulitis.

(d) bacteremia.

8. Vibrio alginolyticus should be considered as an etiologic agent of otitis media in which of the following?

(a) College student vacationing at Daytona Beach (b) Fish cutter from New England

(c) Chesapeake Bay fisherman

(d) All of the above

9. Which of the following is NOT a manifestation of Vitamin A intoxication?

(a) Hypoprothrombinemia

(b) Hypercalcemia

(c) Myocardial hypertrophy (d) Pseudotumor cerebri

10. Retinol binding protein is important in Vitamin A metabolism because

(a) it protects normal tissue from free Vitamin A by binding retinol.

(b) it transports Vitamin A from the intestinal mucosa to the liver.

(c) it esterifies Vitamin A with long chain fatty acides for transport in chylomicrons.

(d) it delivers Vitamin A from the hepatic Ito cell directly to the nucleus of the target cell.

11. The resistance of Hemophilus influenzae type $\mathrm{b}$ to both ampicillin and chloramphenicol is rare throughout the world.

(a) True

(b) False 